\title{
Synthesis and Characterization of Conducting Polymer
}

\author{
Deepak A Zatale ${ }^{1}$, Satish T. Rathod ${ }^{2}$, Kalpana N. Pawar ${ }^{3}$ \\ ${ }^{1}$ Department of Physics, Govt. College of Engineering, Amravati, Maharashtra, India \\ ${ }^{2}$ Department of Mathematics, Shri. Maturadas Mohata Science College, Nagpur, Maharashtra, India \\ ${ }^{3}$ Department of Mathematics, Shri. R. R. Lahoti Science College, Morshi, Amravati, Maharashtra, India
}

\begin{abstract}
Article Info

Volume 8, Issue 3

Page Number : 113-116

\section{Publication Issue}

May-June-2021

\section{Article History}

Accepted : 07 May 2021

Published : 13 May 2021

In recent technology, considerable attention was given to the fabrication of light weight rechargeable batteries, electro chromic display devices, microelectronics, sensor and molecule design etc. As one of the most important conducting polymers, polyaniline because of its chemical stability and relatively high conductivity and its derivatives have been extensively studied in different fields of science, because of the demand for high performance materials in advanced technologies. However, the common uses of polyaniline are restricted, due to its poor process ability and low solubility. Various techniques were given for synthesis of conducting polymer. In the current studies, polyaniline (PANI) and its composites with semiconductor was prepared chemical oxidation method in the presence of different bronsted acids from aqueous solutions. The effect of thermal treatment on electrical conductivity (DC), of the pure PANI, PANI $+10 \%, 15 \%$ and $20 \% \mathrm{MnSO}_{4}$ conducting polymers were investigated. It is found that conductivity of PANI enhancing due to stretching polymeric chain cause due to interaction with $\mathrm{MnSO}_{4}$
\end{abstract}

Keywords : Conducting polymer, DC Conductivity

\section{INTRODUCTION}

In the last two decades the field of conducting polymers has shown tremendous growth and it is now an important field of research, the conducting polymer when functionalized with other conjugate system can be very useful; in many applications. As seen in the literature survey the functionalization with macrocyclic molecules has been mainly used in electrode preparation for reduction of oxygen, the detailed study on functionalization of conducting polymers such as polyaniline with $\mathrm{MnSO}_{4}$ is now reported so far. In order to have better understanding of physics of this material the present work was undertaken. The investigation are mainly clamed at looking at the structural changes taking place due to incorporation of $\mathrm{MnSO}_{4}$ and the effect of $\mathrm{MnSO}_{4}$ on properties such as electrical conductivity, frequency response, and dielectric constant of the resulting polymer composite. In the present investigation, one 
method for synthesizing the conducting polymer composite has been employed. The polymer composite obtained from the method are expected to he thermally stable due to presence of $\mathrm{MnSO}_{4}$. In the doped state these are expected to give higher conductivity because of enhance conjugation level. It is also interesting to see the structural modification imported in the product because of addition of $\mathrm{MnSO}_{4}$.

\section{EXPERIMENTAL}

\section{Electrical Conductivity:}

Electrical conductivity of the synthesized conducting polymer was measured by resistivity measurement technique. The resistance in the pellet form prepared under the hydraulic pressure was done by conventional method. There are two methods generally employed for resistance measurement of the sample. a) Four probe method. b) Two probe method or LCR technique.

TABLE AND GRAPH:

1) Pure PANI $r=0.997 \mathrm{~cm} \quad t=0.47 \mathrm{~cm} \quad A=3.14 \mathrm{~cm}^{2}$ $\mathrm{V}=5$ volts

\begin{tabular}{|c|c|c|c|c|}
\hline $\begin{array}{l}\text { Tem } \\
\mathrm{p}^{0} \mathrm{~K}\end{array}$ & $\begin{array}{c}\text { Curren } \\
\mathrm{t} \mu \mathrm{A}\end{array}$ & $\begin{array}{c}\text { Resistanc } \\
\mathrm{e} \Omega{ }^{*} 10^{6}\end{array}$ & $\begin{array}{c}\text { Specific } \\
\text { resistivit } \\
y \\
(\rho) \\
\Omega \mathrm{cm} \\
{ }^{*} 10^{8}\end{array}$ & $\begin{array}{c}\text { Conductivit } \\
\begin{array}{c}\mathrm{y} \\
\sigma \\
\mathrm{cm}^{-1 *} 10^{-8}\end{array}\end{array}$ \\
\hline 290 & 0.420 & 11.9047 & 1.2573 & 55.213 \\
\hline 310 & 0.435 & 11.4942 & 1.3022 & 59.1223 \\
\hline 320 & 0.455 & 10.9890 & 1.3621 & 60.738 \\
\hline 300 & 0.470 & 10.6382 & 1.4070 & 62.44146 \\
\hline 330 & 0.495 & 10.1010 & 1.4818 & 64.8634 \\
\hline 340 & 0.515 & 9.7087 & 1.5417 & 67.8634 \\
\hline 350 & 0.535 & 9.3457 & 1.6015 & 71.0732 \\
\hline 360 & 0.550 & 9.0990 & 1.646 & 73.4160 \\
\hline 370 & 0.565 & 8.84955 & 1.6914 & 76.39311 \\
\hline 380 & 0.605 & 8.26244 & 1.811 & 79.5544 \\
\hline
\end{tabular}

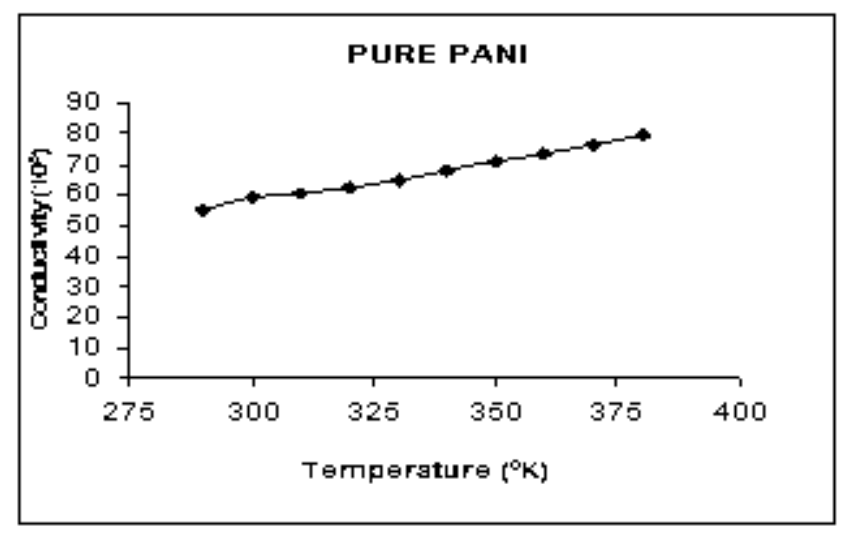

2) $\mathrm{PANI}+10 \% \mathrm{MnSO}_{4} \quad \mathrm{r}=0.996 \mathrm{~cm} . \quad \mathrm{t}=0.313 \mathrm{~cm}$

\begin{tabular}{|c|c|c|c|c|}
\hline $\begin{array}{c}\text { Tem } \\
\mathrm{p}^{\circ} \mathrm{K}\end{array}$ & $\begin{array}{c}\text { Curren } \\
\mathrm{t} \mu \mathrm{A}\end{array}$ & $\begin{array}{c}\text { Resistanc } \\
\text { e } \\
\Omega^{*} 10^{6}\end{array}$ & $\begin{array}{c}\text { Specific } \\
\text { resistivit } \\
y \\
\text { (p) } \Omega \mathrm{Cm} \\
{ }^{*} 10^{6}\end{array}$ & $\begin{array}{l}\text { Conductivit } \\
\qquad \begin{array}{c}\text { y } \sigma \\
\mathrm{S} \mathrm{cm}^{-1 *} 10^{-7}\end{array}\end{array}$ \\
\hline 290 & 0.910 & 5.4945 & 3.363 & 2.9734 \\
\hline 310 & 0.980 & 5.1020 & 3.122 & 3.2022 \\
\hline 320 & 15.90 & 3.1446 & 19.2479 & 5.1922 \\
\hline 300 & 21.8 & 2.2935 & 14.038 & 7.1231 \\
\hline 330 & 22.0 & 2.7272 & 13.911 & 7.1885 \\
\hline 340 & 22.5 & 2.2222 & 13.6019 & 7.3519 \\
\hline 350 & 23.0 & 2.1739 & 13.3062 & 7.5152 \\
\hline 360 & 24.0 & 2.0833 & 12.7517 & 7.8420 \\
\hline 370 & 24.8 & 2.0162 & 12.3404 & 8.1020 \\
\hline 380 & 25.5 & 1.9607 & 12.0016 & 8.3323 \\
\hline
\end{tabular}

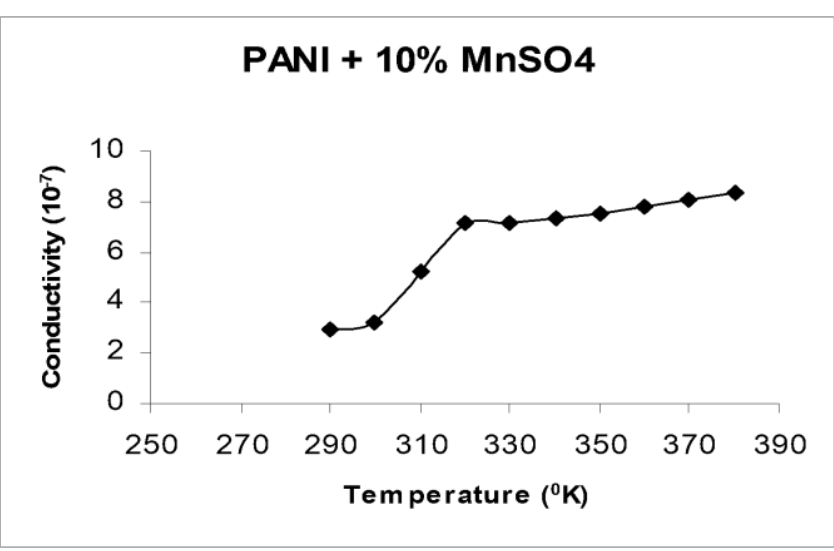


3) $\mathrm{PANI}+15 \% \mathrm{MnSO}_{4} \mathrm{r}=0.998 \mathrm{~cm} \cdot \mathrm{t}=0.490 \mathrm{~cm}$. $\mathrm{A}=3.14 \mathrm{~cm}^{2}$

\begin{tabular}{|c|c|c|c|c|}
\hline $\begin{array}{l}\text { Tem } \\
\mathrm{p}^{0} \mathrm{~K}\end{array}$ & $\begin{array}{c}\text { Curren } \\
\mathrm{t} \mathrm{mA}\end{array}$ & $\begin{array}{c}\text { Resistanc } \\
\text { e } \\
\Omega^{*} 10^{3}\end{array}$ & $\begin{array}{c}\text { Specific } \\
\text { resistivit } \\
\text { y }(\rho) \\
\Omega \mathrm{Cm} \\
{ }^{*} 10^{3}\end{array}$ & $\begin{array}{l}\text { Conductivit } \\
\qquad \begin{array}{c}\text { y } \sigma \\
\mathrm{cm}^{-1 *} 10^{-4}\end{array}\end{array}$ \\
\hline 290 & 12.90 & 0.387 & 2.483 & 4.0261 \\
\hline 300 & 14.45 & 0.340 & 2.217 & 4.5098 \\
\hline 310 & 14.90 & 0.3355 & 2.1503 & 4.4650 \\
\hline 320 & 15.35 & 0.3257 & 2.0873 & 4.7901 \\
\hline 330 & 16.80 & 0.2976 & 1.9071 & 5.2433 \\
\hline 340 & 17.40 & 0.2873 & 1.8414 & 5.4305 \\
\hline 350 & 18.00 & 0.2777 & 1.7800 & 5.6178 \\
\hline 360 & 18.95 & 0.2638 & 1.6908 & 5.9143 \\
\hline 370 & 19.95 & 0.2506 & 1.6060 & 6.2264 \\
\hline 380 & 20.25 & 0.2469 & 1.5822 & 6.3206 \\
\hline
\end{tabular}

\begin{tabular}{|l|c|c|c|c|}
\hline 300 & 154.7 & 0.032 & 0.2510 & 3.9808 \\
\hline 330 & 172.5 & 0.028 & 0.2252 & 4.4388 \\
\hline 340 & 183.5 & 0.0272 & 0.2177 & 4.7219 \\
\hline 350 & 195.4 & 0.0255 & 0.1988 & 5.0281 \\
\hline 360 & 200.9 & 0.0249 & 0.1934 & 5.1696 \\
\hline 370 & 218.3 & 0.0229 & 0.1780 & 5.6174 \\
\hline 380 & 229.9 & 0.0217 & 0.1690 & 5.9158 \\
\hline
\end{tabular}

\section{RESULT}

From the above graph it is indicates that the conductivity of pure polyaniline with $\mathrm{H}_{2} \mathrm{SO}_{4}$ is explained by hopping mechanism other graphs conductivity of polymers now follows very well. So conductivity of polymer is explained by polaron, biopolarons i.e. hoping mechanism.

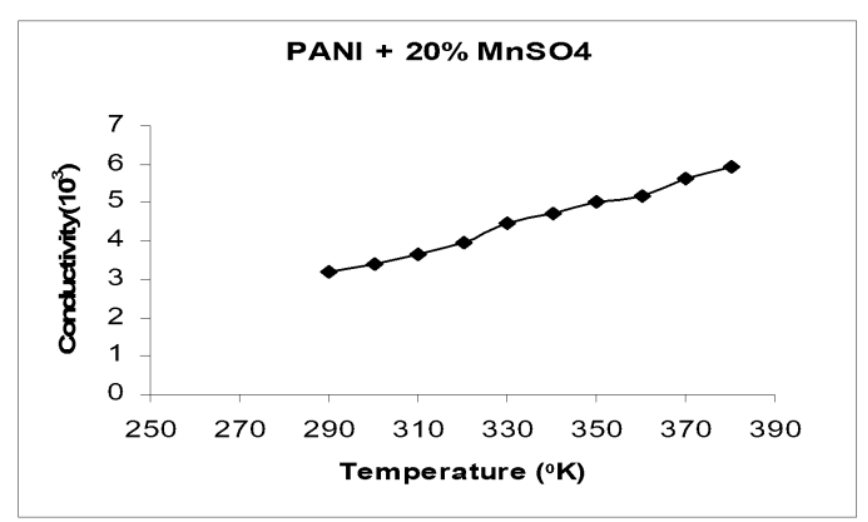

4) $\mathrm{PANI}+20 \% \mathrm{MnSO}_{4} \mathrm{r}=0.997 \mathrm{~cm} . \quad \mathrm{t}=0.404 \mathrm{~cm}$.

$\mathrm{A}=3.14 \mathrm{~cm}^{2}$

\begin{tabular}{|c|c|c|c|c|}
\hline $\begin{array}{c}\text { Temp } \\
{ }^{0} \mathrm{~K}\end{array}$ & $\begin{array}{c}\text { Current } \\
\mathrm{mA}\end{array}$ & $\begin{array}{c}\text { Resistanc } \\
\text { e } \\
\Omega^{*} 10^{3}\end{array}$ & $\begin{array}{c}\text { Specific } \\
\text { resistivit } \\
y \\
(\rho) \\
\Omega \mathrm{Cm} \\
{ }^{*} 10^{3}\end{array}$ & $\begin{array}{c}\text { Conductivi } \\
\text { ty } \\
\sigma \\
\mathrm{cm}^{-1 *} 10^{-3}\end{array}$ \\
\hline 290 & 124.0 & 0.040 & 0.313 & 3.1934 \\
\hline 310 & 132.4 & 0.037 & 0.2935 & 3.4069 \\
\hline 320 & 142.1 & 0.035 & 0.2734 & 3.6565 \\
\hline
\end{tabular}

As the composition $\mathrm{MnSO}_{4}$ is added in pure PANI with $\mathrm{H}_{2} \mathrm{SO}_{4}$ the conductivity of a compound increases. This increase in conductivity shows that the compound become more stretched as compare to pure PANI. As the composition \% increases in PANI nature of graph tells that the semiconductor nature arises in compound. As the temp increases, composite PANI shows the semiconductors enhancing conducting property.

\section{CONCLUSION}

From the above studies we have observed that the value of D.C. \& A.C. conductivity unusually enhanced by doping polymerization of polyaniline. 
Again these conductivity enhanced by polymerization with $\mathrm{MnSO}_{4}$ semiconductor powder. The enhancing conductivity has been attributed due to stretching polymeric chain cause due to interfacial interaction with $\mathrm{MnSO}_{4}$ crystallides. This conductivity in pure PANI and its composites is due to hopping mechanism.

\section{REFERENCES}

[1]. H.C.Pant, M.K.Patra, S.C.Negi,A Bhatia S.R. Vadera \& N Kumar Bull. Mater Sci. 29 (2006) 379.

[2]. Polymer engineering science. M.Blaszkiewicz, D.S. Mchlachian

[3]. S.Radhakrushanan and A.K Arof, Polymeric Materials,Naro, Publ.Hou.(1998).

[4]. R Murugesan and E Subramanian Bull. Mater Sci. 26 (2003) 529.

[5]. C O Yoon, M Reghu, D Moses, Y Cao and A J Heeger Synth. Met. 26 (1995) 255.

[6]. J Joo, S M Long, J P Pouget, E J Oh, A G MacDiarmid and A Epstein, Phys. Rev. B 57(1995) 67.7)

[7]. I. Harada, Y.Furukawa and F Ueda Synth. Met.29 (1998) 303. B.Wessling Synth. Met 102 (1999) 1396.

[8]. J Yang, J Hou,W Zhu, Xu and M Wan Synth. Met. 80 (1996) 283.

[9]. An introduction to polymer science. Hans By Georg Elias

[10]. polymer science By Gowarikar

[11]. Polymer and advanced material. A.G. MacDiarmic \& A Epstein

\section{Cite this article as :}

Deepak A Zatale, Satish T. Rathod, Kalpana N. Pawar, "Synthesis and Characterization of Conducting Polymer", International Journal of Scientific Research in Science and Technology (IJSRST), Online ISSN : 2395-602X, Print ISSN : 2395-6011, Volume 8 Issue 3, pp. 113-116, May-June 2021. Available at doi : https://doi.org/10.32628/IJSRST218328 Journal URL : https://ijsrst.com/IJSRST218328 